\title{
Efficacy of chemotherapy plus gefitinib treatment in advanced non-small-cell lung cancer patients following acquired resistance to gefitinib
}

\author{
ZHENGBO SONG ${ }^{1,2}$ and YIPING ZHANG ${ }^{1,2}$ \\ ${ }^{1}$ Department of Chemotherapy, Zhejiang Cancer Hospital; \\ ${ }^{2}$ Key Laboratory Diagnosis and Treatment Technology on Thoracic Oncology, Hangzhou, Zhejiang 310022, P.R. China
}

Received February 14, 2013; Accepted July 9, 2013

DOI: $10.3892 / \operatorname{mco} .2013 .156$

\begin{abstract}
Non-small-cell lung cancer (NSCLC) may exhibit oncogene addiction in patients who benefited from prior treatment with epidermal growth factor receptor (EGFR)-tyrosine kinase inhibitors (TKIs). Preclinical data suggested that EGFR addiction persists after the development of TKI resistance, leading many clinicians to continue TKI treatment along with chemotherapy. However, this strategy has not been adequately evaluated in clinical practice. Patients who benefited from gefitinib followed by acquired resistance to this drug were reviewed in the Zhejiang Cancer Hospital. Patients were included if they received chemotherapy and gefitinib following failure of prior gefitinib treatment. A total of 26 patients were included in the present study. Six patients (23.1\%) exhibited a partial response (PR), 13 (50\%) achieved stable disease (SD) and 7 (26.9\%) had progressive disease (PD) during the chemotherapy and gefitinib treatment. The disease control rate (DCR) was $73.1 \%$ and the median progression-free survival (PFS) was 4.6 months [95\% confidence interval (CI): 3.8-5.4]. The toxicities associated with gefitinib and chemotherapy were generally acceptable. In conclusion, continued concurrent gefitinib and chemotherapy may be a valuable strategy, with acceptable and well-tolerated toxicity. However, this treatment requires further investigation.
\end{abstract}

\section{Introduction}

Treatment with the epidermal growth factor receptor (EGFR) tyrosine kinase inhibitors (TKIs) gefitinib and erlotinib has

Correspondence to: Dr Yiping Zhang, Department of Chemotherapy, Zhejiang Cancer Hospital, 38 Guangji Road, Hangzhou, Zhejiang 310022, P.R. China

E-mail: yipingzhang@yahoo.cn

Key words: non-small-cell lung cancer, gefitinib, combination, efficacy led to significant clinical improvement in certain patients with advanced non-small-cell lung cancer (NSCLC), particularly those of Asian descent, non-smokers and those with adenocarcinoma (1-4).

Despite prolonged survival, it should be noted that discontinuation of EGFR inhibition may cause more rapid progression of symptoms and lesions in certain patients, which is referred to as 'disease flare' (5). The most likely explanation for this phenomenon is oncogene addiction, which is recognized in several types of cancer. Gastrointestinal stromal tumors have a unique biology and exhibit rapid disease progression when the kinase inhibitor imatinib is removed after prolonged benefit (6). This series describes a similar flare phenomenon in the setting of acquired resistance in EGFR-mutant lung cancer when gefitinib or erlotinib are discontinued due to disease progression.

An effective treatment for patients with disease flare has not yet been established. Preclinical studies indicated that continuous TKI administration may be a valuable strategy. However, available published data on the clinical activity of gefitinib combination chemotherapy following failure of gefitinib are limited. Therefore, the role of combination treatment after gefitinib failure remains remains debatable. This study was retrospectively performed to evaluate the role of combination treatment following gefitinib failure in patients with advanced NSCLC.

\section{Patients and methods}

Patients. This retrospective study was conducted through a review of medical records of patients with advanced NSCLC who received gefitinib combined with chemotherapy following disease progression due to gefitinib failure, between July, 2010 and June, 2012. The study was approved by the Ethics Committee of the Zhejiang Cancer Hospital. Eligibility criteria included: i) histological or cytological diagnosis of stage IIIb or IV NSCLC; ii) at least one measurable tumor lesion; iii) initial gefitinib treatment for $>6$ months and acquired resistance to gefitinib according to Jackman's criteria (7); and iv) discontinuation time between the prior treatment and re-administration of gefitinib of $\leq 1$ week. The characteristics of the study population are shown in Table I. 
Table I. Baseline characteristics of the study population $(n=26)$.

\begin{tabular}{|c|c|c|}
\hline Variables & No. & Percentage \\
\hline \multicolumn{3}{|l|}{ Gender } \\
\hline Male & 14 & 53.8 \\
\hline Female & 12 & 46.2 \\
\hline \multicolumn{3}{|l|}{ PS } \\
\hline $0-1$ & 13 & 50.0 \\
\hline 2 & 13 & 50.0 \\
\hline \multicolumn{3}{|l|}{ Age } \\
\hline Median & 56 & \\
\hline Mean & 57 & \\
\hline$<65$ & 18 & 69.2 \\
\hline$\geq 65$ & 8 & 30.8 \\
\hline \multicolumn{3}{|l|}{ Smoking status } \\
\hline Yes & 9 & 34.6 \\
\hline No & 17 & 65.4 \\
\hline \multicolumn{3}{|l|}{ Chemotherapy } \\
\hline Pemetrexed & 14 & 53.8 \\
\hline Docetaxel & 12 & 46.2 \\
\hline \multicolumn{3}{|l|}{ Stage } \\
\hline IIIb & 0 & 0 \\
\hline IV & 26 & 100 \\
\hline \multicolumn{3}{|l|}{ Histology } \\
\hline Adenocarcinoma & 22 & 84.6 \\
\hline Non-adenocarcinoma & 4 & 15.4 \\
\hline $\begin{array}{l}\text { Median duration of prior } \\
\text { gefitinib treatment }\end{array}$ & \multicolumn{2}{|c|}{9.6 months } \\
\hline
\end{tabular}

PS, performance status.

Methods. Patients were administered gefitinib orally from day 1 of the first cycle and pemetrexed or docetaxel as an intravenous (i.v.) infusion on day 1 . Pemetrexed was administered as a $10-\mathrm{min}$ i.v. infusion and docetaxel $75 \mathrm{mg} / \mathrm{m}^{2}$ as a 1 -h i.v. infusion once every 3 weeks. Chemotherapy (pemetrexed or docetaxel) was discontinued if no progression occurred at the end of 4 cycles and gefitinib was continuously administered until disease progression.

Evaluation of response and toxicity. The tumor response was classified in accordance with the Response Evaluation Criteria in Solid Tumors (RECIST) 1.1. The patients were evaluated to determine the stage of their disease prior to treatment initiation and at the time of disease progression or relapse, by computed tomography (CT) of the chest and abdomen and other staging procedures. Adverse events were evaluated according to the Common Terminology Criteria for Adverse Events (CTCAE) 3.0.

Statistical methods. Kaplan-Meier survival curves were used to estimate overall survival (OS) and progression-free survival (PFS). OS was measured from the first day of combination

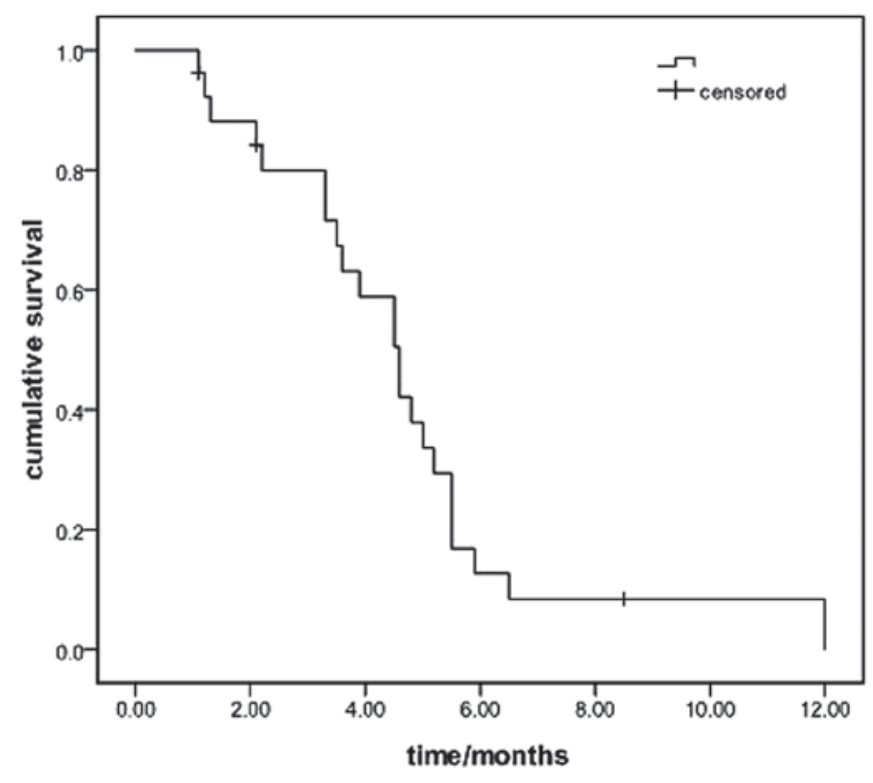

Figure 1. Progression-free survival in the 26 patients.

treatment to the day the patient succumbed or last follow-up. PFS was defined as the interval from the initiation of combination treatment to treatment failure or the date of the last follow-up. All the analyses were performed with SPSS software version 16 (SPSS Inc., Chicago, IL, USA).

\section{Results}

Patient characteristics. A total of 26 patients (14 males and 12 females) were included in the present study. The median age of the patients was 56 years (range, $42-71$ years). The performance status (PS) score was $0-1$ in 13 patients $(50 \%)$ and 2 in the remaining $50 \%$. The majority of tumors $(84.6 \%)$ were adenocarcinomas with advanced stage at presentation and $34.6 \%(9 / 26)$ of the patients had a history of smoking. The median duration of the initial gefitinib treatment was 9.6 months [95\% confidence interval (CI): 7.5-12.0]. Docetaxel was administered to 12 and pemetrexed to 14 patients, concurrently with gefitinib treatment.

Response data and survival analysis. The median follow-up period for the 26 patients was 8.0 months (range, 1.0-15 months). Sixteen patients had exhibited a PR and 10 had SD during the prior gefitinib treatment. The response to combination treatment included 6 cases of PR, 13 of SD and 7 of progressive disease (PD), which accounted for a disease control rate (DCR) of $73.1 \%$. The median PFS was 4.6 months (95\% CI: 3.8-5.4; Fig. 1). The median OS of the entire patient sample was 7.3 months (95\% CI: 6.1-8.5 months) (Fig. 2). Of the 26 patients, 13 underwent analysis of EGFR mutations and 10 were found to harbor activating mutations, including 6 patients with exon 19 deletions and 4 with exon 21 L858R mutations, whereas 3 had a negative mutational status. The median PFS of the 10 patients harboring EGFR mutation was 4.6 months, with 4.2 months for the EGFR wild-type patients $(\mathrm{P}=0.86)$.

Prognostic factors. In the univariate analysis, PS had a statistically significant effect on PFS (Table II). No significant 
Table II. Univariate analysis of PFS in the 26 patients.

\begin{tabular}{llll}
\hline Variables & PFS & $95 \%$ CI & P-value \\
\hline Gender & & & 0.013 \\
Male & 4.5 & $2.9-6.3$ & \\
Female & 4.6 & $4.2-6.8$ & \\
Age (years) & & & 0.57 \\
$\geq 65$ & 4.5 & $3.2-6.4$ & \\
$<65$ & 4.8 & $1.3-7.7$ & \\
PS & & & 0.043 \\
0-1 & 5.5 & $5.2-5.8$ & \\
2 & 3.3 & $1.9-4.7$ & \\
Chemotherapy & & & \\
Pemetrexed & 3.9 & $1.5-6.3$ & \\
Docetaxel & 4.6 & $3.9-5.3$ & \\
Smoking history & & & \\
Yes & 4.6 & $3.4-5.8$ & \\
No & 5.2 & $4.0-6.4$ & \\
Histology & & & \\
Non-adenocarcinoma & 3.3 & $1.1-6.4$ & \\
Adenocarcinoma & 4.6 & $4.2-5.0$ & \\
Treatment line & & & \\
Third-line & 4.6 & $1.1-8.1$ & \\
Further-line & 4.5 & $3.3-5.7$ & \\
\hline
\end{tabular}

PFS, progression-free survival; CI, confidence interval; PS, performance status.

differences in PFS were observed with respect to other factors. The Cox regression model was constructed with the incorporation of age, gender, histological grade, smoking history, chemotherapeutic regimen and PS. PS was identified as the only independent prognostic factor $(\mathrm{P}=0.043)$.

Treatment toxicities. Grade 1/2 skin and hematological toxicities were observed in 13 and 18 patients, respectively. Grade 2 diarrhea developed in 6 patients. Toxicities were considered acceptable, with grade $3 / 4$ skin toxicity in 5 and neutropenia in 9 patients. Two patients had a dosage reduction due to grade 4 neutropenia and 2 patients developed hepatic function abnormalities following gefitinib treatment.

\section{Discussion}

In the present study, the response rate and DCR with gefitinib and chemotherapy following failure of gefitinib treatment were 23.1 and $73.1 \%$, respectively. The median PFS was 4.6 months, which was considered to be favorable compared to previous third- or further-line treatments. The outcome indicated that this treatment was an optimal choice for the patients after failure of gefitinib therapy.

According to the guidelines of the National Comprehensive Cancer Network (8), EGFR-TKIs are recommended as a second- or third-line treatment regimen for

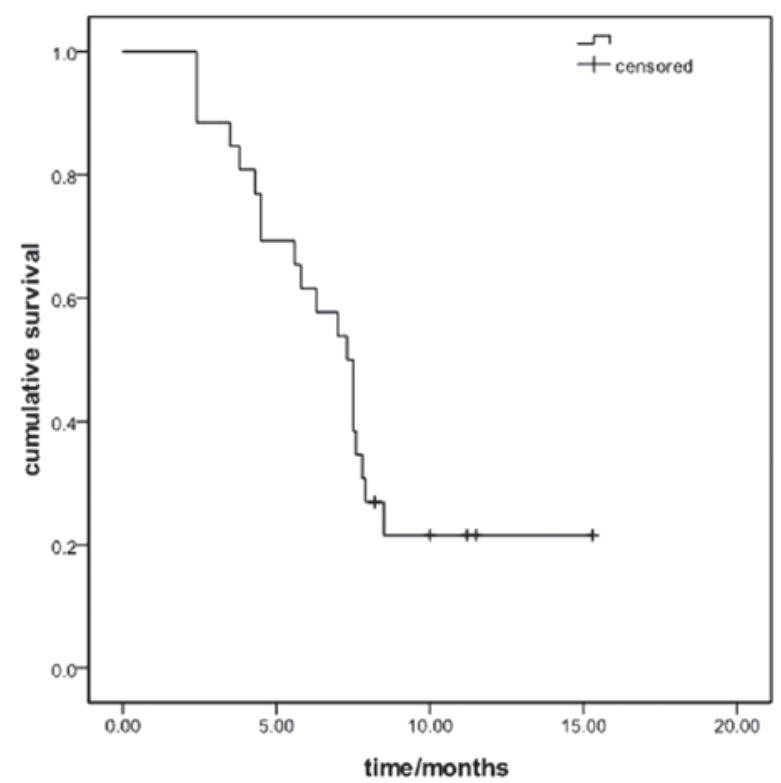

Figure 2. Overall survival in the 26 patients.

patients with NSCLC. However, there were no established treatment protocols for patients following failure of previous gefitinib or erlotinib treatment and discontinuation of EGFR inhibition may cause more rapid progression of symptoms and lesions in certain patients. Chaft et al (5) observed a $23 \%$ flare rate during the EGFR TKI washout period following disease progression under TKI treatment. Therefore, discontinuation of TKI treatment may not be suitable for patients who benefited from gefitinib or erlotinib.

According to an ASCO 2012 retrospective study, continuation of erlotinib and chemotherapy following failure of erlotinib treatment enhanced the overall response rate (ORR) and achieved a PFS of 4.4 months [Goldberg et al (9)]. In the present study the ORR was $23.1 \%$, which was similar to that reported by Goldberg et al (9). The outcome indicated that patients may also benefit from the gefitinib combination treatment.

Treatment with gefitinib-pemetrexed or docetaxel was generally well-tolerated and the adverse events (AEs) were similar to those observed in previous studies of each agent alone (10-13). The most common AEs were grade 1/2 skin rash and hematological toxicity.

The present study indicated the efficacy and safety of combined pemetrexed/docetaxel therapy as subsequent treatment in patients with gefitinib-resistant tumors that had exhibited an initial response to gefitinib monotherapy. However, the small sample size of this study may not be sufficient to accurately interpret the study results. Further assessment in a large-scale prospective study is required to obtain definitive evidence. A phase II trial (NCT01707329) has been initiated in our hospital to investigate the efficacy of combination treatment following failure of icotinib, another EGFR-TKI, the efficacy of which was shown to be similar to that of gefitinib in a phase III trial (14).

In conclusion, gefitinib combined with chemotherapy for Chinese patients with advanced NSCLC achieved promising ORR, DCR and PFS, with an acceptable toxicity profile. 


\section{References}

1. Mok TS, Wu YL, Thongprasert S, et al: Gefitinib or carboplatin-paclitaxel in pulmonary adenocarcinoma. N Engl J Med 361: 947-957, 2009.

2. Shepherd FA, Pereira JR, Ciuleanu T, et al: Erlotinib in previously treated non-small-cell lung cancer. N Engl J Med 353: 123-132, 2005.

3. Mitsudomi T, Morita S, Yatabe Y, et al: Gefitinib versus cisplatin plus docetaxel in patients with non-small-cell lung cancer harbouring mutations of the epidermal growth factor receptor (WJTOG3405): an open label, randomised phase 3 trial. Lancet Oncol 11: 121-128, 2010

4. Maemondo M, Inoue A, Kobayashi K, et al: Gefitinib or chemotherapy for non-small-cell lung cancer with mutated EGFR. N Engl J Med 362: 2380-2388, 2010.

5. Chaft JE, Oxnard GR, Sima CS, Kris MG, Miller VA and Riely GJ: Disease flare after tyrosine kinase inhibitor discontinuation in patients with EGFR-mutant lung cancer and acquired resistance to erlotinib or gefitinib: implications for clinical trial design. Clin Cancer Res 17: 6298-6303, 2011.

6. Van den Abbeele AD, Badawi RD, Manola J, et al: Effects of cessation of imatinib mesylate (IM) therapy in patients (pts) with IM-refractory gastrointestinal stromal tumors (GIST) as visualized by FDG-PET scanning. J Clin Oncol 22: abs. 3012, 2004.

7. Jackman D, Pao W, Riely GJ, et al: Clinical definition of acquired resistance to epidermal growth factor receptor tyrosine kinase inhibitors in non-small-cell lung cancer. J Clin Oncol 28: $357-360,2010$.
8. National Comprehensive Cancer Network (NCCN): NCCN Clinical Practice Guidelines in Oncology. Available at: http:// www.nccn.org/index.asp. Accessed: January 4, 2013.

9. Goldberg SB, Oxnard OR, Digumarthy S, Muzikansky A, Jackman DM, Lennes IT and Sequist LV: Chemotherapy with erlotinib or chemotherapy alone in advanced NSCLC with acquired resistance to EGFR tyrosine kinase inhibitors (TKI). J Clin Oncol 30 (Suppl): abs. 7524, 2012.

10. Shepherd FA, Dancey J, Ramlau R, et al: Prospective randomized trial of docetaxel versus best supportive care in patients with non-small-cell lung cancer previously treated with platinum-based chemotherapy. J Clin Oncol 18: 2095-2103, 2000.

11. Hanna N, Shepherd FA, Fossella FV, et al: Randomized phase III trial of pemetrexed versus docetaxel in patients with non-small-cell lung cancer previously treated with chemotherapy. J Clin Oncol 22: 1589-1597, 2004.

12. Kim ES, Hirsh V, Mok T, et al: Gefitinib versus docetaxel in previously treated non-small-cell lung cancer (INTEREST): a randomised phase III trial. Lancet 22: 1809-1818, 2008.

13. Maruyama R, Nishiwaki Y, Tamura T, Yamamoto N, et al: Phase III study, V-15-32, of gefitinib versus docetaxel in previously treated Japanese patients with non-small-cell lung cancer. J Clin Oncol 26: 4244-4252, 2008.

14. Sun Y, Shi Y, Zhang L, et al: A randomized, double-blind phase III study of icotinib versus gefitinib in patients with advanced non-small cell lung cancer (NSCLC) previously treated with chemotherapy (ICOGEN). J Clin Oncol 29 (Suppl 15): abs. 7522, 2011. 